\title{
Approach to first-order exact solutions of the Ablowitz-Ladik equation
}

\author{
Adrian Ankiewicz, ${ }^{1}$ Nail Akhmediev, ${ }^{1}$ and Falk Lederer ${ }^{2}$ \\ ${ }^{1}$ Optical Sciences Group, Research School of Physics and Engineering, Institute of Advanced Studies, \\ The Australian National University, Canberra ACT 0200, Australia \\ ${ }^{2}$ Institute of Condensed Matter Theory and Solid State Optics, Friedrich-Schiller-Universität Jena, \\ Max-Wien-Platz 1, D-07743 Jena, Germany
}

(Received 24 December 2010; published 4 May 2011)

\begin{abstract}
We derive exact solutions of the Ablowitz-Ladik (A-L) equation using a special ansatz that linearly relates the real and imaginary parts of the complex function. This ansatz allows us to derive a family of first-order solutions of the A-L equation with two independent parameters. This novel technique shows that every exact solution of the A-L equation has a direct analog among first-order solutions of the nonlinear Schrödinger equation (NLSE).

DOI: 10.1103/PhysRevE.83.056602

PACS number(s): 05.45.Yv, 45.05.+x, 42.81.Qb, 42.65.Wi
\end{abstract}

\section{INTRODUCTION}

Extreme waves, for example, optical needles, can occur in spatially extended systems. Recently, such waves have been observed in a nonlinear optical cavity made with a ring oscillator with a liquid crystal light valve as the gain medium [1].

Discrete dynamical systems are often applied to model arrays of optical wave guides [2], electronic circuits [3,4], nonlinear lattices [5], and a variety of other structures in physics and engineering.

The Ablowitz-Ladik equation is one of the basic discrete equations of the integrable hierarchy. As such it serves as a model for the application of a range of techniques for obtaining exact solutions. The Ablowitz-Ladik equation is one of the early examples of an equation to which the inverse scattering technique has been applied. Various other methods, such as Darboux and Backlund transformations, have also been applied to solve this equation. Generally speaking, every technique applicable to the basic NLSE equation can also be applied, with some modifications, to the A-L equation. These modifications are far from being trivial, since discrete and continuous variables have to be treated differently. Nevertheless, the concept is there.

Discrete systems have specific features that make them more difficult to solve than continuous differential equations. Even integrable equations such as the A-L equation present certain problems. The difficulties do appear [6] despite the fact that the inverse scattering technique for this system is well known. Thus, any new technique that allows us to obtain solutions is of interest and will present new properties of solutions, thus strengthening our abilities to deal with the equation. Moreover, deriving a class of solutions using a new technique means that this class has hidden symmetries that were unknown and are waiting to be revealed. Clearly, a new technique is of great value by itself, even if particular solutions of this class were previously obtained using other methods.

One of the ways to solve the continuous NLSE is using the ansatz that was first applied to the NLSE by Akhmediev and Korneev (the AK ansatz) [7]. It was used later in [8] to find the whole family of first-order solutions of the self-focusing NLSE (see also the book [9] for more detailed derivations). Our question here is as follows: Can the same ansatz be used to solve the A-L equation? The answer turns out to be in the affirmative. Below, we present a few examples of solutions derived this way.

The standard form of the integrable A-L equation can be written as [10-13]:

$$
i \frac{\partial \psi_{n}}{\partial t}+\left(\psi_{n-1}+\psi_{n+1}\right)\left(1+\left|\psi_{n}\right|^{2}\right)-2 \psi_{n}=0
$$

where $t$ is the continuous evolution variable (time or longitudinal spatial variable) and $n=0, \pm 1, \pm 2, \ldots$ are integers.

\section{LINEAR ANSATZ}

The complex function $\psi_{n}(t)$ on a constant background of amplitude $q$ can be written explicitly as

$$
\psi_{n}(t)=\left[R_{n}(t)-q+i J_{n}(t)\right] e^{i 2 q^{2} t+i \phi_{0}},
$$

where $R_{n}(t)$ and $J_{n}(t)$ are real functions. The NLS equation can be solved with a simple ansatz which was first used in the work [7]. This ansatz leads to a rich family of first-order solutions of the NLSE. A whole class of solutions was thus derived from first principles. Our conjecture here is that a corresponding ansatz can be used for the A-L equation (1). Namely, we propose that the real and imaginary parts of the solution in the complex plane, rotating with the angular frequency $2 q^{2}$, are linearly related, that is, for arbitrary $n$ we can write

$$
J_{n}(t)=m(t) R_{n}(t)+c(t)
$$

where the coefficients $m(t)$ and $c(t)$ depend only on $t$. In this paper, we first consider a subset of this class, viz. solutions additionally having the property that $c(t)=0$. Later, in Sec. VI, we present the analysis with $c(t) \neq 0$.

Let us use (3) and substitute (2) into (1). Namely, from (3), we have

$$
J_{n}^{\prime}(t)=m^{\prime}(t) R_{n}(t)+m(t) R_{n}^{\prime}(t) .
$$

After the substitution, separating the real and imaginary parts of the A-L equation we obtain two equations:

$$
\begin{aligned}
& b\left(R_{n-1}+R_{n+1}-2 q\right)+2 q\left(1+q^{2}\right) \\
& \quad-R_{n}\left(2+2 q^{2}+m^{\prime}(t)\right)-m R_{n}^{\prime}=0,
\end{aligned}
$$

and

$$
m b\left(R_{n-1}+R_{n+1}\right)+R_{n}^{\prime}-2\left(1+q^{2}\right) m R_{n}=0,
$$


where

$$
b=\left[1+q^{2}-2 q R_{n}\left(1+m^{2}\right) R_{n}^{2}\right] .
$$

Now, we insert $R_{n-1}+R_{n+1}$ from Eq. (5) into Eq. (6) and get a first-order differential equation in $R_{n}(t)$. We simplify this further by defining

$$
P_{n}(t)=1 / R_{n}(t) .
$$

Then we obtain the ordinary differential equation for $P_{n}(t)$ :

$$
P_{n}^{\prime}(t)=2 q m(t)+\frac{m(t)\left[m^{\prime}(t)-4 q^{2}\right]}{1+m^{2}(t)} P_{n}(t) .
$$

Solving this equation allows us to find the function $m(t)$ and hence exact solutions of the A-L equation itself. This can be done in a few steps.

\section{EXACT SOLUTION FOR MODULATION INSTABILITY (MI)}

To obtain the MI solution, we assume that variables $n$ and $t$ can be separated, so that Eq. (8) has a solution in the form of a product:

$$
P_{n}(t)=s_{1}+s_{2} f(n) g(t),
$$

where $s_{1}>0$, and $s_{2}$ are real constants but $f(n)(\neq$ const. $)$ is a function of $n$. Then Eq. (8) gives

$$
A(t)-f(n) B(t)=0,
$$

where

$$
A(t)=2 q-4 s_{1} q^{2}+2 q m^{2}(t)+s_{1} m^{\prime}(t)
$$

and

$$
B(t)=\frac{s_{2}}{m(t)}\left[\left(1+m^{2}(t)\right) g^{\prime}(t)+g(t) m(t)\left(4 q^{2}-m^{\prime}(t)\right)\right] .
$$

Clearly, $A(t)$ and $B(t)$ in (9) must both be zero. Thus, solving $A(t)=0$ [with $m(0)=0$ ], we obtain

$$
m(t)=\sqrt{2 s_{1} q-1} \tanh \left[\frac{2 q}{s_{1}} \sqrt{2 s_{1} q-1} t\right] .
$$

Then, using Eq. (10) and solving $B(t)=0$ [with $g(0)=$ $1, g^{\prime}(0)=0$ ] shows that

$$
g(t)=\operatorname{sech}\left(\frac{2 q}{s_{1}} \sqrt{2 s_{1} q-1} t\right) .
$$

We define

$$
s_{1}=\frac{q}{2 v\left(1+q^{2}\right)},
$$

so now the argument of the hyperbolic functions is $\delta t$ where

$$
\delta=4 \sqrt{\left(1+q^{2}\right) v\left[q^{2}(1-v)-v\right]} .
$$

Thus, we now have the functions of $t$ and now only need to find the function of $n$.

We define $v=\sin ^{2}(\kappa / 2)$ for convenience. The effect of modulation instability requires that we must have an oscillatory function of $n$ for the transverse direction. The phase of this function is not important. For simplicity, we take an even function, $f(-n)=f(n)$ and set $f(n)=\cos (\kappa n)$. Here $\kappa$ is an arbitrary value in the MI range, that is, $0<\kappa<\kappa_{\max }$, where

$$
\kappa_{\max }=\arccos \left(\frac{1-q^{2}}{1+q^{2}}\right) .
$$

Taking $n=t=0$ in Eq. (5) shows that

$$
s_{2}=\frac{c_{1}}{2 v\left(1+q^{2}\right)},
$$

where

$$
c_{1}=\sqrt{\frac{q^{2}(1-v)-v}{1-v}} .
$$

Finally, we have

$$
P_{n}(t)=\frac{q \pm c_{1} \cos (\kappa n) \operatorname{sech}(\delta t)}{2 v\left(1+q^{2}\right)} .
$$

Thus, having the solution expression for $P_{n}(t)$, we easily derive the solution of the A-L equation:

$$
\psi_{n}(t)=\left[\frac{2\left(1+q^{2}\right) v+i \frac{\delta}{2} \tanh (\delta t)}{q \pm c_{1} \cos (\kappa n) \operatorname{sech}(\delta t)}-q\right] e^{i 2 q^{2} t+i \phi},
$$

where $\kappa$ belongs to the interval of instability. The solution can also be written in the form:

$\psi_{n}(t)=e^{2 i q^{2} t}\left[\frac{2\left(1+q^{2}\right) v \cosh (\delta t)+i \frac{\delta}{2} \sinh (\delta t)}{q \cosh (\delta t) \pm c_{1} \cos (\kappa n)}-q\right]$.

The period in the transverse $(n)$ direction is $2 \pi / \kappa$. The solution in this form has been presented earlier in [12]. It can also be written in other forms [13]. From the form (13), it can be seen that this solution is a complete discrete analog of the NLSE solution (38) given in the work [8]. This can be seen more clearly if we combine the two terms in (13) into one:

$$
\begin{aligned}
\psi_{n}(t)= & e^{2 i q^{2} t} \\
& \times \frac{\left(2 v+2 v q^{2}-q^{2}\right) \cosh (\delta t) \mp c_{1} \cos (\kappa n)+i \frac{\delta}{2} \sinh (\delta t)}{q \cosh (\delta t) \pm c_{1} \cos (\kappa n)} .
\end{aligned}
$$

Apart from the constant coefficients, all functional terms in this solution are the same as in (38) of [8]. This shows that we can establish a one-to-one correspondence between the solutions of the A-L and NLSE equations. The values $q$ and $\kappa$ are two independent parameters of the family of solutions defined by Eq. (14). Two more parameters can be introduced as translations along the $t$ and $n$ axes. While translations along the $t$ axis seem to be trivial, as they only shift the solution by an arbitrary $t_{0}$ in the $t$ direction, the shifts along the $n$ axis are more complicated. When the shift $n_{0}$ is an integer, the solution is indeed just shifted by an integer number of nodes. When $n_{0}$ is not an integer, the shift produces a new solution that has different values at the nodes.

If, for $\kappa$, we choose the point of the maximum growth rate, $\kappa_{0}=\arccos \left(\frac{1}{1+q^{2}}\right)$, then the dependent parameters will take 


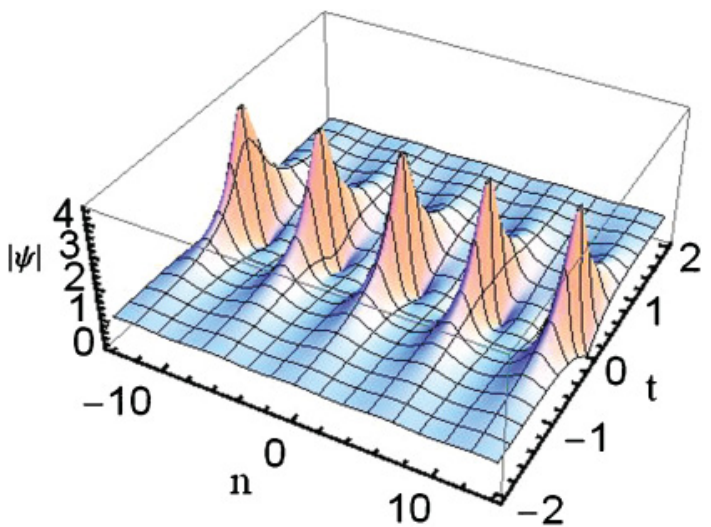

FIG. 1. (Color online) Transversely periodic solution of the A-L equation given by Eq. (14). The evolution starts with a constant background $q$, slightly modulated, then the modulation increases to reach its maximum at $t=0$ and finally the solution returns back to the original background, $q$. Here, $q=1$ and $\kappa=\frac{\pi}{3}$.

the following values: $\delta=2 q^{2}, v=\frac{q^{2}}{2\left(1+q^{2}\right)}$, and $c_{1}=q \sqrt{\frac{1+q^{2}}{2+q^{2}}}$. In this special case, the solution can be reduced to a simpler form:

$\Psi_{n}(t)=i q e^{2 i q^{2} t}\left[\frac{\sqrt{2+q^{2}} \sinh \left(2 q^{2} t\right) \mp i \sqrt{1+q^{2}} \cos \left(\kappa_{0} n\right)}{\sqrt{2+q^{2}} \cosh \left(2 q^{2} t\right) \pm \sqrt{1+q^{2}} \cos \left(\kappa_{0} n\right)}\right]$,

where $\kappa_{0}$ is fixed, as given above. In this form, we have a complete analogy with the Akhmediev Breather (AB) solution (39) of the work [8]. This solution is illustrated in Fig. 1. Here, we have also fixed $q=1$. This choice provides an integer value for the period along the $n$ axis. When the shift $n_{0}$ is zero, the maximum values of the soluton are located at discrete sites.

\section{SOLITON ON A BACKGROUND}

To obtain the soliton solution on a background, we take the constant part of $P_{n}$ to be negative. This seems like a minor change, but it effectively swaps trigonometric and hyperbolic functions, and thus changes the solution from being periodic in the transverse $(n)$ direction to being periodic in the propagation $(t)$ direction. Thus, physically, the soliton case is quite different from MI. We take background as $q$, which is an arbitrary number. We set

$$
P_{n}(t)=s_{3}\left[-1 \pm s_{5} f(n) g(t)\right]
$$

where $s_{3}>0, s_{5}$ are constants but $f(n)(\neq$ const. $)$ is a function of $n$. Then Eq. (8) gives

$$
A(t)+f(n) B(t)=0
$$

where

$$
A(t)=2 q m(t)-\frac{s_{3} m(t)}{1+m^{2}(t)}\left(m^{\prime}(t)-4 q^{2}\right)=0,
$$

and

$$
B(t)=\frac{s_{3} s_{5} g(t) m(t)}{1+m^{2}(t)}\left(m^{\prime}(t)-4 q^{2}\right)-s_{3} s_{5} g^{\prime}(t) .
$$

Solving $A(t)=0$ [with $m(0)=0$ ], we obtain

$$
m(t)=\sqrt{1+2 q s_{3}} \tan \left[\frac{2 q \sqrt{1+2 q s_{3}}}{s_{3}} t\right] .
$$

Then, using Eq. (17) and solving $B(t)=0$ [with $g^{\prime}(0)=0$ ] shows that

$$
g(t)=\sec \left(\frac{2 q \sqrt{1+2 q s_{3}}}{s_{3}} t\right) .
$$

In light of the swap of trigonometric and hyperbolic functions, we can expect $f(n)$ to be the hyperbolic analog of cos, viz. cosh. We thus set $f(n)=\cosh \left(\kappa_{2} n\right)$, with $\kappa_{2}$ having an arbitrary value. We define

$$
u=\sinh \left(\kappa_{2} / 2\right)
$$

for convenience, so that $\kappa_{2}=2 \operatorname{arcsinh}(u)$. This shows that the solution is aperiodic in the transverse direction. On substitution, we find

$$
s_{3}=\frac{q}{2\left(q^{2}+1\right) u^{2}} \quad \text { and } \quad s_{5}=\sqrt{\frac{1+\left(1+q^{-2}\right) u^{2}}{1+u^{2}}} .
$$

Finally, the slope $m(t)$ can be rewritten as $m(t)=s_{6} \tan \left(w_{1} t\right)$, where

$$
s_{6}=\sqrt{1+\frac{q^{2}}{\left(q^{2}+1\right) u^{2}}}
$$

and

$$
w_{1}=4\left(q^{2}+1\right) u \sqrt{u^{2}+\frac{q^{2}}{q^{2}+1}} .
$$

Thus, in this formulation, $q$ and $u$ are the arbitrary parameters. Once given, we can then find $s_{3}, s_{5}, s_{6}$, and $w_{1}$.

As a result, we have the following expression for $P_{n}(t)$ :

$$
P_{n}(t)=s_{3}\left[-1 \pm s_{5} \cosh \left(\kappa_{2} n\right) \sec \left(w_{1} t\right)\right] .
$$

Using $R_{n}(t)=1 / P_{n}(t)$ and Eq. (3), this leads to the solution of the A-L equation in its simplest form:

$$
\psi_{n}(t)=e^{2 i q^{2} t}\left[\frac{1+i s_{6} \tan \left(w_{1} t\right)}{s_{3}\left[-1 \pm s_{5} \cosh \left(\kappa_{2} n\right) \sec \left(w_{1} t\right)\right]}-q\right] .
$$

The period in the propagation $(t)$ direction, $2 \pi / w_{1}$ decreases from $\infty$ to 0 as $u$ increases from 0 to $\infty$. Thus all frequencies are possible, in contrast to the modulation instability case of Sec. III. For $u \rightarrow 0$, we recover the first-order rogue discrete solution. This solution can also be written in the form:

$$
\begin{aligned}
\psi_{n}(t)= & e^{2 i q^{2} t} \\
& \times \frac{\left(1+q s_{3}\right) \cos \left(w_{1} t\right) \mp q s_{3} s_{5} \cosh \left(\kappa_{2} n\right)+i s_{6} \sin \left(w_{1} t\right)}{-s_{3} \cos \left(w_{1} t\right) \pm s_{3} s_{5} \cosh \left(\kappa_{2} n\right)} .
\end{aligned}
$$

This solution is a direct discrete analog of the NLSE solution (52) of the work [8]. The latter is known as a soliton on a 


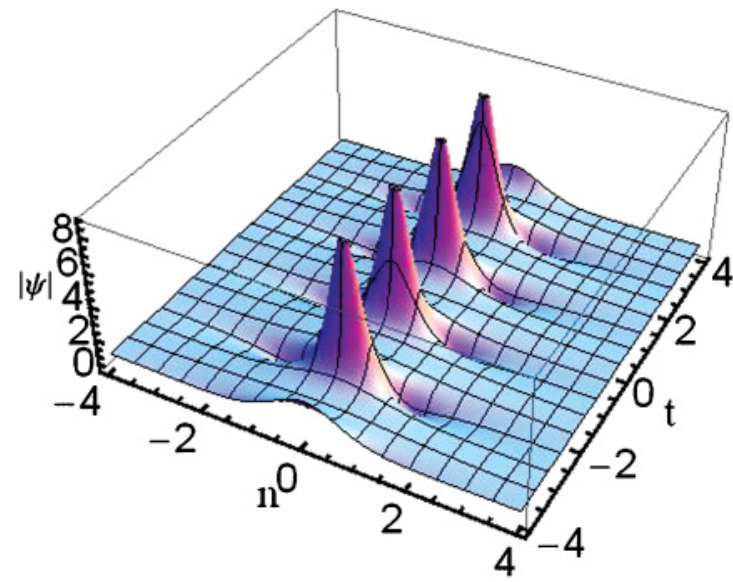

FIG. 2. (Color online) Soliton on a background defined by Eqs. (18) and (19). Here, $q=1$ and $\kappa_{2}=1$.

background or "Ma soliton." An illustrative example is shown in Fig. 2.

\section{FUNDAMENTAL ROGUE WAVE}

The way to obtain this is simply to take a sum of the functions (instead of a product):

$$
P_{n}(t)=r_{1}+f(n)+g(t),
$$

where $r_{1}$ is a constant but $f(n)$ is a function of $n$ with $f(0)=0$. Then Eq. (8) gives

$$
A_{1}(t)-f(n) B_{1}(t)=0 .
$$

The functions $A_{1}(t)$ and $B_{1}(t)$ must both be zero. Now

$$
B_{1}(t)=m^{\prime}(t)-4 q^{2}=0 .
$$

Thus, with $m(0)=0$, we have

$$
m(t)=4 q^{2} t .
$$

Then $A_{1}(t)=0$ means that $g^{\prime}(t)=8 q^{3} t$, so, with $g(0)=0$,

$$
g(t)=4 q^{3} t^{2}
$$

We need a function which is even in variable $n$ for $f(n)$ [so that $f(-n)=f(n)]$. Substituting into the recurrence relation, Eq. (5) with $n=0$ shows that $r_{1}=\frac{1}{4 q\left(1+q^{2}\right)}$ and $f(1)=\frac{q}{1+q^{2}}$; then $n=1$ gives $f(2)=4 \frac{q}{1+q^{2}}$, while $n=2$ gives $f(3)=$ $9 \frac{q}{1+q^{2}}$, etc. We obtain $f(n)=\frac{q}{1+q^{2}}[0,1,4,9,16,25,36, \ldots]$, for $n=[0,1,2,3,4, \ldots]$, that is, $f(n)=\frac{q n^{2}}{1+q^{2}}$. Thus we have derived all the required functions.

Hence,

$$
P_{n}=\frac{1}{1+q^{2}}\left[\frac{1}{4 q}+n^{2} q\right]+4 q^{3} t^{2}
$$

Inverting this function provides the real part of $\psi_{n}$ :

$$
R_{n}(t)=\frac{1}{P_{n}(t)}=\frac{4 q\left(1+q^{2}\right)}{1+4 n^{2} q^{2}+16 q^{4}\left(1+q^{2}\right) t^{2}} .
$$

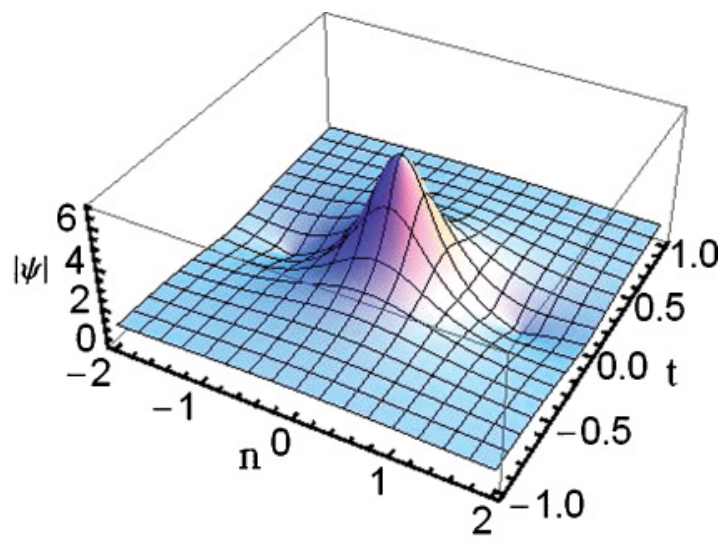

FIG. 3. (Color online) Rational solution of the first order defined by Eq. (21). Here, $q=1$. The maximum amplitude is 7 .

Thus, the solution can be written in the form:

$$
\begin{aligned}
\psi_{n}(t)= & e^{i 2 q^{2} t+i \phi} \\
& \times q\left[\frac{4\left(1+q^{2}\right)\left(1+4 i q^{2} t\right)}{1+4 q^{2} n^{2}+16 q^{4}\left(1+q^{2}\right) t^{2}}-1\right],
\end{aligned}
$$

which is a rational solution of the A-L equation [14]. Furthermore, this is a discrete analog of the solution (40) in the work [8] which is presently known as "Peregrine soliton" $[15,16]$. It is illustrated in Fig. 3. As this solution is localized in both the $t$ and $n$ directions, it can be called "a wave that appears from nowhere and disappears without a trace" [17]. In case of the NLSE, it serves as a prototype of a rogue wave in the deep ocean [16,18-22]. Its existence in optical fibers has been demonstrated in a recent experimental work [23].

\section{SOLUTIONS PERIODIC IN $\boldsymbol{n}$ AND $\boldsymbol{t}$.}

The existence of homoclinic and heteroclinic orbits considered above means that there are periodic ones around them. As the system is completely integrable, the saddle points with modulation instability have nearby hyperbolic trajectories that continue to these periodic orbits. In order to find them, we now use

$$
\psi_{n}(t)=\left[G_{n}(t)+i J_{n}(t)\right] e^{i \omega t+i \phi},
$$

where $\omega=2 k_{b} q^{2}$, and, as before, we suppose that $G_{n}(t)$ and $J_{n}(t)$ are linearly related:

$$
J_{n}(t)=m(t) G_{n}(t)+c(t) .
$$

As there is no explicitly separable background for periodic solutions, we have generalized (3) and effectively included $q$ in the term $G_{n}(t)$.

On substituting (22) into Eq. (1), as before, we separate real and imaginary parts and take the sum $G_{n-1}(t)+G_{n+1}(t)$ from one of them and substitute into the other. This way, we find

$$
\begin{gathered}
2 c(t)\left[c^{2}(t)+2 c(t) G_{n} m(t)-q^{2} k_{b}\right]+m(t)\left[c^{\prime}(t)+G_{n} m^{\prime}(t)\right] \\
+\left[1+m^{2}(t)\right]\left[\frac{d G_{n}}{d t}+2 c(t) G_{n}^{2}\right]=0 .
\end{gathered}
$$


For convenience, we set $m(t)=c(t) \hat{m}(t)$, and use

$$
1 / G_{n}(t)=\frac{f(n)}{g(t)}-\hat{m}(t) .
$$

Upon substitution, we find

$$
D(t)+A(t) f^{2}(n)+B(t) f(n)=0 .
$$

Clearly, each component must be zero separately:

$$
\begin{gathered}
D(t)=\hat{m}^{\prime}(t)-2 c(t)\left[q^{2} k_{b} \hat{m}^{2}(t)-1\right]=0, \\
A(t)=c(t)\left[2 c^{2}(t)-2 q^{2} k_{b}+\hat{m}(t) c^{\prime}(t)\right]=0,
\end{gathered}
$$

and

$$
\begin{aligned}
B(t)= & c(t) g(t) \hat{m}(t)\left[4 q^{2} k_{b}-\hat{m}(t) c^{\prime}(t)\right]+g^{\prime}(t) \\
& +\hat{m}(t) c^{2}(t)\left[\hat{m}(t) g^{\prime}(t)+\hat{m}^{\prime}(t) g(t)\right]=0 .
\end{aligned}
$$

Solving Eqs. (26) and (27), we obtain $\hat{m}(t)$ and $c(t)$ as single functions:

$$
\hat{m}(t)=\frac{\sqrt{k_{b}}}{q} \operatorname{cd}\left(s t, k_{b}^{2}\right),
$$

and

$$
c(t)=q \sqrt{k_{b}} \operatorname{sn}\left(s t, k_{b}^{2}\right),
$$

where sn and cd are Jacobi elliptic functions with moduli of $m_{b}=k_{b}^{2}$, while the coefficient $s=2 q^{2}$. There are two different definitions for moduli of Jacobi elliptic functions in the literature. One of them is simply the square of the other. For clarity, here, we are using the convention that produces the series $\operatorname{sn}(t, y)=t-t^{3}(1+y) / 6+\ldots$ Using these, we solve $B(t)=0$ and obtain the remaining function of $t$ :

$$
g(t)=q \sqrt{k} \operatorname{dn}\left(s t, k_{b}^{2}\right) .
$$

We now need only the single function of $n$, and this turns out to be

$$
f(n)=k_{t} \mathrm{nc}\left(r n, k^{2}\right),
$$

where $r$ is arbitrary and $k_{t}$ is an unknown constant.

If we define $c_{m}=\operatorname{cn}\left(r, k^{2}\right)$, then $k_{b}=\sin (\theta)$, where

$$
\tan (\theta)=\frac{1}{k \sqrt{1-k^{2}}}\left(\frac{1}{1+c_{m}}-k^{2}\right) .
$$

Then

$$
q^{2}=\frac{k \sqrt{1-k^{2}} s_{m}^{2}}{\sqrt{1-k_{b}^{2}} c_{m}}
$$

where $s_{m}=\operatorname{sn}\left(r, k^{2}\right)$. This constant agrees with that found in [24].

This leads to

$$
G_{n}(t)=q \sqrt{k} \frac{\operatorname{dn}\left(s t, k_{b}^{2}\right) \operatorname{cn}\left(r n, k^{2}\right)}{k_{t}-\sqrt{k k_{b}} \operatorname{cn}\left(s t, k_{b}^{2}\right) \operatorname{cn}\left(r n, k^{2}\right)},
$$

and hence the complete solution,

$$
\begin{aligned}
\psi_{n}(t)= & q e^{i \omega t+i \phi} \\
& \times \frac{\sqrt{k} \operatorname{dn}\left(s t, k_{b}^{2}\right) \operatorname{cn}\left(r n, k^{2}\right)+i \sqrt{k_{b}} k_{t} \operatorname{sn}\left(s t, k_{b}^{2}\right)}{k_{t}-\sqrt{k k_{b}} \operatorname{cn}\left(s t, k_{b}^{2}\right) \operatorname{cn}\left(r n, k^{2}\right)},
\end{aligned}
$$

where $k_{t}^{4}=\left(1-k^{2}\right)\left(1-k_{b}^{2}\right)$. This solution can be considered a discrete analog of Eq. (45) in the work [8]. It has been derived in a quite different way in [24].

In the limit $k \rightarrow 0$ and $k_{b} \rightarrow 1, q$ becomes the background amplitude and $m(t) \rightarrow \tanh \left(2 q^{2} t\right)$. Then $r=\kappa_{0}=$ $\arccos \left(1 /\left(1+q^{2}\right)\right)=k_{1 g}$. The period along the $t$ axis then becomes infinite and the solution, Eq. (28), reduces to

$$
\psi_{n}(t)=q \frac{\cos (r n)+i \frac{k_{t}}{\sqrt{k}} \sinh \left(2 q^{2} t\right)}{\frac{k_{t}}{\sqrt{k}} \cosh \left(2 q^{2} t\right)-\cos (r n)} e^{i 2 q^{2} t+i \phi} .
$$

Clearly,

$$
\frac{k_{t}}{\sqrt{k}}=\sqrt{\frac{2+q^{2}}{1+q^{2}}},
$$

so Eq. (29) agrees with Eq. (15).

In the opposite limiting case, $k \rightarrow 0$, we obtain the soliton solution from Eq. (28).

\section{CONCLUSION}

We have derived first-order exact solutions of the AblowitzLadik equation using the $\mathrm{AK}$ ansatz that linearly relates the real and imaginary parts of the solution. Each of the solutions has a corresponding solution of the NLS equation, so related physical phenomena can occur in systems governed by these two equations.

\section{ACKNOWLEDGMENTS}

Two of the authors (A.A. and N.A.) acknowledge the support of the Australian Research Council (Discovery Project No. DP0985394). N.A. is a grateful recipient of support from the Alexander von Humboldt Foundation (Germany).
[1] A. Montina, U. Bortolozzo, S. Residori, and F. T. Arecchi, Phys. Rev. Lett. 103, 173901 (2009).

[2] A. B. Aceves, C. De Angelis, T. Peschel, R. Muschall, F. Lederer, S. Trillo, and S. Wabnitz, Phys. Rev. E 53, 1172 (1996).

[3] P. Marquie, J. M. Bilbault, and M. Remoissenet, Phys. Rev. E 51, 6127 (1995).

[4] J. M. Bilbault and P. Marquie, Phys. Rev. E 53, 5403 (1996).

[5] D. Hennig and G. P. Tsironis, Phys. Rep. 307, 333 (1999).
[6] P. D. Miller, Commun. Pure Appl. Math. 48, 1369 (1995).

[7] N. N. Akhmediev and V. I. Korneev, Teor. Mat. Fiz. 69, 189 (1986); [Theor. Math. Phys. (USSR) 69, 1089 (1986)].

[8] N. N. Akhmediev, V. M. Eleonskii, and N. E. Kulagin, Teor. Mat. Fiz. 72, 809 (1986) [Theor. Math. Phys. (USSR) 72, 183 (1987)].

[9] N. Akhmediev and A. Ankiewicz, Solitons (Chapman and Hall, London, 1997). 
[10] M. J. Ablowitz and J. F. Ladik, Stud. Appl. Math. 55, 213 (1976).

[11] M. J. Ablowitz and J. F. Ladik, J. Math. Phys. 17, 1011 (1976).

[12] N. Akhmediev and A. Ankiewicz, Phys. Rev. E 83, 046603 (2011).

[13] A. Ankiewicz, N. Akhmediev, and J. M. Soto-Crespo, Phys. Rev. E 82, 026602 (2010).

[14] K. Narita, J. Phys. Soc. Jpn. 59, 3528 (1990).

[15] D. H. Peregrine, J. Aust. Math. Soc. Series B 25, 16 (1983).

[16] V. I. Shrira and V. V. Geogjaev, J. Eng. Math. 67, 11 (2010).

[17] N. Akhmediev, A. Ankiewicz, and M. Taki, Phys. Lett. A 373, 675 (2009).

[18] K. B. Dysthe and K. Trulsen, Phys. Scr. T82, 48 (1999).

[19] V. V. Voronovich, V. I. Shrira, and G. Thomas, J. Fluid Mech. 604, 263 (2008).
[20] I. Ten and H. Tomita, "Simulation of the ocean waves and appearance of freak waves," Reports of RIAM Symposium No. 17SP1-2, Proceedings of a symposium held at Chikushi Campus, Kyushu University, Kasuga, Fukuoka, Japan, March 10-11, 2006.

[21] D. Clamond, M. Francius, J. Grue, and C. Kharif, European J. Mech. B - Fluids 25, 536 (2006).

[22] N. Akhmediev and E. Pelinovsky (editors), "Rogue wavestoward a unifying concept," Special Issue of Eur. Phys. J. Special Topics 185 (2010).

[23] B. Kibler, J. Fatome, C. Finot, G. Millot, F. Dias, G. Genty, N. Akhmediev, and J. M. Dudley, Nature Phys. 6, 790 (2010)

[24] K. Chow, R. Conte, and N. Xu, Phys. Lett. A 349, 422 (2006). 